\title{
Descriptive and Clinical Epidemiology of Cholera in Al-Hudaydah, Yemen
}

\author{
Abdulla S. Bin Ghouth ${ }^{1} \quad$ Ghanim Y.M. Alsheikh ${ }^{2}$ \\ 1.Professor of Community Medicine \\ Hadramout University College of Medicine (HUCOM), Mukalla, Yemen \\ 2.WHO Collaborating Center for Public Health Education and Training, Imperial College London, UK. \\ Former Dean, Hadramout University College of Medicine, Mukalla, Yemen
}

\begin{abstract}
Background: Yemen where a new cholera outbreak started in 2016 and continued to spread till 2020, becoming the largest documented cholera epidemic of modern times. Al-Hudaydah at western part of Yemen was the most affected governorates in Yemen by cholera outbreak that considered about $15 \%$ of all cases in Yemen. The aim of this study is to fill the gap in the knowledge about the clinical epidemiology of cholera outbreak in AlHudaydah in Yemen in 2018. Methods: This is a retrospective analysis of secondary surveillance data spanning between January 1st and December 31, 2018 from Al-Hudaydah governorate at western part of Yemen. Results: A total of 13819 patients with acute watery diarrhea were reported in three diarrhea treatment centers in AlHudaydah governorate in 2018. The median age is 13 years, about one third of the patients are under 5 years of age (34.4\%). The peak of the epidemic was occurred in the epi weeks 40 to 50 corresponding to the period from half of September to half of December 2018. most of them had some dehydration (66.6\%) About $24.1 \%$ had no dehydration while only $9.3 \%$ of patients had severe dehydration. Only 1618 Rapid diagnostic tests (RDT) were positive for cholera (24\%) while vibrio cholera was isolated from $97.6 \%$ of 1438 stool specimens from RDTs positive patients. Only $18 \%$ of cholera patients were admitted to in-patient department of the DTCs. It was reported very low death rate $(0.1 \%)$. The treatment regimen used for patient care are vary from ORS only $(45 \%)$ to ORS and IV fluid (36.2\%) or sometimes accompanied fluid therapy with antibiotic. Conclusions: There is no doubt the AWD occurred in Yemen is cholera; Even CFR may be underestimated, good performance of DTCs reduce the case fatality rate from cholera among severely dehydrated patients to less than $1 \%$
\end{abstract}

Keywords: Cholera, Al-Hudaydah, Yemen.

DOI: $10.7176 / \mathrm{JHMN} / 80-04$

Publication date:September $30^{\text {th }} 2020$

\section{Background}

Cholera is an acute watery diarrheal disease caused by the ingestion of food or water contaminated with the toxigenic strains of Vibrio cholerae serogroups O1 or O139 [1]. Cholera is often characterized by watery diarrhea, with or without vomiting, and severe dehydration, resulting in death if left untreated [2]. Vibrio cholerae produces a profuse watery diarrhea that can quickly progress to dehydration and hypovolemic shock, and can kill up to $50 \%$ of patients if fluids are not properly replaced. [3] However, the case fatality rate (CFR) can be less than 1\% with appropriate case management. [1] Fluid and electrolyte replacement is the mainstay of treatment of cholera patients; the severe cases require antibiotic treatment to reduce the duration of illness and replacement of fluid intake. The antibiotic therapy currently has faced difficulties due to the rapid emergence and spread of multidrug resistant V. cholerae causing several outbreaks in the globe. Currently, cholera has been becoming endemic in an increasing number of geographical areas, reflecting a failure in implementation of control measures. [4]

The seventh cholera pandemic has caused a large burden of disease worldwide for more than 50 years, with the highest burden of disease in Africa, the Americas and southern Asia. Explosive cholera outbreaks have been observed in the past 10 years in several African countries and Haiti. Seventh pandemic strains have spread in the Middle East causing outbreaks in several countries, including Yemen where a new cholera outbreak started in 2016 and continued to spread till 2020, becoming the largest documented cholera epidemic of modern times. [1] The current cholera epidemic in Yemen, linked to spread of $V$. cholerae O1 (Ogawa serotype), is associated with the ongoing war. [5] Al-Hudaydah governorate at western part of Yemen is one of the most affected governorates (of 3,200,00 population) in Yemen by cholera outbreak. in this governorate there were 163500 cholera cases were reported during the period Between Sept 28, 2016, and March 12, 2018 [ [6] that considered about $15 \%$ of all cases in Yemen at that time. In 2018 cholera outbreak continue in spread especially in AlHudaydah governorate; up to my knowledge nothing was writing about the clinical epidemiology of cholera outbreak in Al-Hudaydah in 2018. So, the aim of this study is to filling the gap in the knowledge about the clinical epidemiology of cholera outbreak in Al-Hudaydah in Yemen in 2018. 


\section{Methods}

This is a retrospective analysis of secondary surveillance data spanning between January 1st and December 31, 2018 from Al-Hudaydah governorate at western part of Yemen. The surveillance system in Yemen used the syndrome approach for cholera and given the name of Acute Watery Diarrhea (AWD).

In response to the cholera outbreak in Yemen, a case definition of a suspected case of AWD was developed and clearly stated that: any patient presenting with three or more liquid stools with or without vomiting in the past $24 \mathrm{~h}$. A confirmed case was a suspected case with $V$ cholerae O1 or O139 confirmed by culture. This database contains dates of disease onset and admission, age, sex, district of origin, dehydration severity (based on the World Health Organization "WHO" severity grading), [] ] laboratory results when available, and disease outcome (discharge or death). District surveillance officers compiled the line-list from all health facilities in their district. This district line-list database was sent electronically to the governorate level each day. Data were aggregated by the Emergency Operation Centre run by the Yemen Health Authorities and cleaned by WHO surveillance officers to remove duplicates, standardized district names, and solve inconsistent entries (e.g. inverted month and day in date format). [6]

\section{Results}

A total of 13819 patients with acute watery diarrhea (AWD) were reported in three DTCs in Al-Hudaydah governorate in 2018 . The mean age of patients is 18.4 years $( \pm 18)$ and the median age is 13 years (interquartile range is 25), age scope shows great variations, the range from one month to 100 years. About one third of the patients are under 5 years of age (34.4\%) while patients at 60 years and above are $5.2 \%$. Most of the cases $(99 \%)$ occurred in just three districts. Zabid and Bait Al-Faqeh at the south part of the governorate $(38.4 \%$ and $23.9 \%$ respectively) and Al-Hali district in Al-Hudaydah city (36.7\%). All patients were reported in the three diarrhea treatment centers DTCs; Zabid health center (38.4\%) Al-Salkhanah hospital in the city 36.7\%) and Bait AlFaqeh hospital (23.9\%). See table 1.

Table 1. Socio-demographic characteristics of cholera patients

\begin{tabular}{|l|l|l|l|}
\hline \multicolumn{2}{|l|}{ Characteristics } & No of patients (N-13819) & $\%$ \\
\hline \multirow{4}{*}{ Age group } & $0-4$ years & 4758 & $34.4 \%$ \\
\cline { 2 - 4 } & $5-14$ years & 2627 & $19 \%$ \\
\cline { 2 - 4 } & $15-29$ years & 3131 & $22.7 \%$ \\
\cline { 2 - 4 } & $30-49$ years & 2589 & $18.7 \%$ \\
\cline { 2 - 4 } & $60+$ years & 712 & $5.2 \%$ \\
\hline \multirow{5}{*}{ Sex } & Male & 7202 & $52.1 \%$ \\
\cline { 2 - 4 } & Female & 6617 & $47.9 \%$ \\
\hline \multirow{5}{*}{ Districts } & Zabid & 5303 & $38.4 \%$ \\
\cline { 2 - 4 } & Al-Hali & 5074 & $36.7 \%$ \\
\cline { 2 - 4 } & Bait Al-Faqeh & 3303 & $23.9 \%$ \\
\cline { 2 - 4 } & Other districts & 139 & $1 \%$ \\
\hline \multirow{5}{*}{ The reporting DTC } & Zabid health center & 5137 & $38.5 \%$ \\
\cline { 2 - 4 } & Al-Salkhanah hospital & 5151 & $37.3 \%$ \\
\cline { 2 - 4 } & Bait Al-Faqeh hospital & 3351 & $24.2 \%$ \\
\hline
\end{tabular}

The epidemic curve of cholera:

Figure 1 shows that the peak of the epidemic occurred in the Epidemiological week (Epi Weeks) from 40 to 50 corresponding to the period from half of September to half of December 2018. See figure 1. 


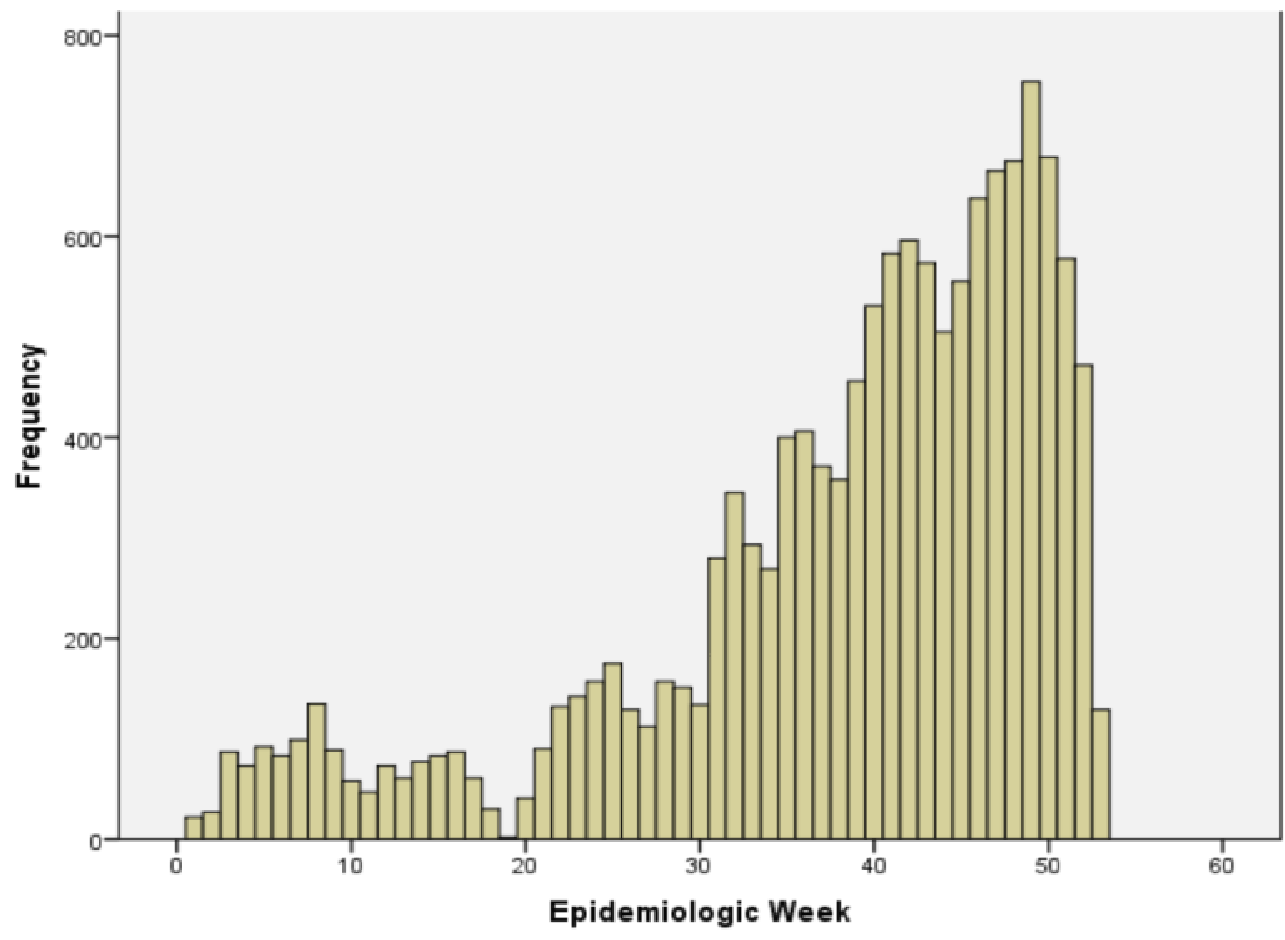

Figure 1. The Epidemic Curve of Cholera in Al-Hudaydah governorate, Yemen in 2018

\section{Clinical presentation, treatment and outcome}

While all patients had diarrhea, only 55\% had vomiting and most of them had some dehydration (66.6\%) About $24.1 \%$ had no dehydration while only $9.3 \%$ of patients had severe dehydration.

Rapid diagnostic test (RDT) specific for cholera were used for 6714 patients while only 1618 tests were positive for cholera (24\%). When 1438 stool specimens from RDTs positive patients were investigated by culture for vibrio cholera, the culture result shows that in 1403 cultures vibrio cholera were isolated (97.6\%). Table 2

Only $18 \%$ of cholera patients were admitted to in-patient department of the DTCs. It was reported very low death rate $(0.1 \%)$. The treatment regimen used for patient care are vary from ORS only $(45 \%)$ to Oral rehydration solution (ORS) and intravenous fluid (IV fluid) (36.2\%) or sometimes accompanied fluid therapy with antibiotic. See table 2.

Table 2. Clinical presentation. Treatment regimen and outcomes

\begin{tabular}{|c|c|c|c|}
\hline \multicolumn{2}{|c|}{ Clinical Characteristics } & Patients (N-13819) & $\%$ \\
\hline \multirow{2}{*}{ Vomiting } & Yes & 7597 & $55 \%$ \\
\hline & No & 6222 & $45 \%$ \\
\hline \multirow[t]{3}{*}{ Dehydration } & No dehydration & 3333 & $24.1 \%$ \\
\hline & Some dehydration & 9197 & $66.6 \%$ \\
\hline & Severe dehydration & 1289 & $9.3 \%$ \\
\hline \multirow{2}{*}{$\begin{array}{l}\text { Rapid test result } \\
(\mathrm{N}=6714)\end{array}$} & Positive & 1618 & $24 \%$ \\
\hline & Negative & 5096 & $76 \%$ \\
\hline \multirow{2}{*}{$\begin{array}{l}\text { Stool culture result } \\
(\mathrm{N}=1438)\end{array}$} & Positive & 1403 & $97.6 \%$ \\
\hline & Negative & 35 & $2.4 \%$ \\
\hline \multirow{2}{*}{$\begin{array}{l}\text { Admitted to the } \\
\text { DTCs }\end{array}$} & In-patient admission & 2493 & $18 \%$ \\
\hline & Treated in the Oral Rehydration Therapy (ORT) corners & 11326 & $82 \%$ \\
\hline \multirow{4}{*}{$\begin{array}{l}\text { Treatment regimen } \\
\text { used }\end{array}$} & ORS only & 6228 & $45 \%$ \\
\hline & ORS + antibiotic & 198 & $1.5 \%$ \\
\hline & ORS+IV fluid & 5002 & $36.2 \%$ \\
\hline & ORS+IV fluid +antibiotic & 2391 & $17.3 \%$ \\
\hline \multirow{2}{*}{$\begin{array}{l}\text { Outcome } \\
\text { treatment }\end{array}$} & Cure & 13807 & $99.9 \%$ \\
\hline & Died & 12 & $0.1 \%$ \\
\hline
\end{tabular}


The deaths (12 cases) occurred among 1289 severely dehydrated patients giving the CFR among severe dehydration group to $0.93 \%$. while case CFR by age group shows that elder patients are more at risk of death from cholera $(\mathrm{CFR}=0.42 \%)$. See table 3

Table 3. CFR by age group

\begin{tabular}{|l|l|l|l|}
\hline Age group & No of cases & No of deaths & CFR \\
\hline $0-4$ years & 4758 & 2 & $0.04 \%$ \\
\hline 5-14 years & 2627 & 3 & $0.11 \%$ \\
\hline $15-29$ years & 3131 & 2 & $0.06 \%$ \\
\hline $30-49$ years & 2589 & 2 & $0.08 \%$ \\
\hline $60+$ years & 712 & 3 & $0.42 \%$ \\
\hline Total & 13819 & 12 & $0.1 \%$ \\
\hline
\end{tabular}

\section{Discussion}

Access to safe water and improved sanitation facilities has eliminated cholera transmission of Vibrio cholerae, the causative agent, in high-income countries. However, the bacteria continue to afflict millions of people in less developed countries where improved water and sanitation infrastructure are not widely available. [7] This is the case in Yemen with adding the current war consequences on the environment and the infrastructure make multiepidemics and poverty is the title of Yemen including the current waves of cholera.

The actual global burden of cholera is largely unknown as the vast majority of cases are not reported. The World Health Organization (WHO) estimates that only $5-10 \%$ of the cases occurring annually are officially reported [8]. This low reporting efficiency is due to a combination of factors including limited capacity of epidemiological surveillance systems and laboratories, and social, political and economic disincentives for reporting [9-11]. Cholera in Yemen may be present since several years but was not reported that is not means no cholera exist as the current cholera outbreaks appears suddenly in 2016 and continue till now, Cholera outbreak in Al-Hudaydah governorate is an example.

Controversial interpretation of the low CFR in Yemen was reported; Over-reporting of cases has likely contributed to underestimates of the CFR in this epidemic. Additionally, poor access to health facilities, especially in areas near the frontline and directly affected by the war like Al-Hudaydah, could have led to underestimation of cholera burden, notably of mortality, as previously demonstrated in Haiti. [12] This low reporting efficiency is due to a combination of factors However, Médecins Sans Frontières' (MSF) experience in Yemen suggests that the low estimates of the CFR could also reflect high rates of health-care seeking among the Yemeni population. Nearly all patients $(96 \%)$ visiting health facilities with suspected cholera arrived within 2 days of symptom onset.[] ] Unlike in other countries where cholera occurs, the first places people in Yemen visited for care tended to be health facilities taking part in the cholera surveillance system, as opposed to private clinics or non-allopathic healers. Until reliable cholera community death estimates become available for Yemen, CFR estimates should be interpreted with caution. [6] Regarding this issue two explanations for this controversial: first the low CFR is true as all the severe cases were attending the health facilities even from remote areas and the death is naturally occurred among the severe cases. The second explanation; not all AWD cases were true cholera that maximize the denominator and giving underestimation of the CFR. The annual number of cholera deaths for the endemic countries were estimated from the number of cholera cases multiplied by the cholera case-fatality rate. Many deaths occur before these patients are able to reach a health facility. Therefore, it was assumed that facility-based case fatality rates (CFRs) were underestimating the true population CFRs. Instead of using facility-based CFRs, CFRs were calculated using inverse variance-weighted average CFRs by WHO mortality stratum. The sensitivity analysis assumed CFR of cholera from $1 \%$ CFR to $5 \%$ CFR. [13] apply this assumption on the cholera outbreak of Al-Hudaydah in 2018 (with 13819 cases and 12 deaths) the number of cholera related deaths will be within the range of 138 to 691 deaths.

Cholera is often characterized by watery diarrhea, with or without vomiting, and severe dehydration, resulting in death if left untreated. [2] in this study all patients presented with different degree of diarrhea, about $55 \%$ presenting with vomiting and only $9.3 \%$ have severe dehydration. The deaths (12 cases) occurred among 1289 severely dehydrated patients giving the CFR among severe dehydration group to $0.93 \%$. while case CFR by age group shows that elder patients are more at risk of death from cholera $(\mathrm{CFR}=0.42 \%)$. Cholera mortality is not limited to a certain age group, and is high among all patients. Due to the rapid dehydration of the cholera cases. [13]

\section{Conclusion}

There is no doubt the that acute watery diarrhea arisen in Al-Hudaydah, Yemen is cholera. Despite that the Case Fatality Rate is underestimated; good performance of Diarrhea Treatment Centers reduces the case fatality rate from cholera among severely dehydrated patients to less than $1 \%$. 


\section{List of Abbreviations}

AWD: Acute Watery Diarrhea; CFR: Case Fatality Rate; DTCs: Diarrhea Treatment Centers; Epi. Wks.: Epidemiological Weeks; I.V. Fluid.: Intravenous Fluid; MSF: Médecins Sans Frontières; RDT: Rapid Diagnostic Test; ORS: Oral Rehydration Solution; ORT: Oral Rehydration Therapy; WHO: World Health Organization.

\section{Declarations}

Ethics approval and consent to participate: not applicable

Consent for publication: Not applicable

Availability of data and materials: All data set kept confidentiality with the authors.

Competency of interest: Authors declare that there is no conflict of interest.

Funding: No funding agency support this study and all tasks and activities cost were covered from Authors' pockets.

Authors' contribution: Both authors prepared the proposal and formulated the study design. First author collected the secondary data, both authors did the analysis, writing and revised the final report and approve it.

Acknowledgements: Authors thank all who gave advice in proposal development especially deanship of Hadramout University College of Medicine. Thanks also are extended to surveillance staff who facilitated data collection.

\section{References}

1. Clemens JD, Nair GB, Ahmed T, et al. Cholera. Lancet. 2017; 390:1539-49.

2.Microbiology Society. Cholera: Death by Diarrhea: Fact File. 2016. https://microbiologysociety.org/publication/education-outreach-resources/cholera-death-by-diarrhoea.html

3. Phelps M, Perner ML, Pitzer VE, Andreasen V, Jensen PKM, Simonsen L. Cholera epidemics of the past offer new insights into an old enemy. J Infect Dis. 2018; 217: 641-649.

4. Mandal S, Mandal MD, Pal NK. Cholera: a great global concern. Asian Pac J Trop Med. 2011; 4(7):573-580. doi:10.1016/S1995-7645(11)60149-1

5. Rabaan AA. Cholera: an overview with reference to the Yemen epidemic. Front Med. 2019;13(2):213-228. doi:10.1007/s11684-018-0631-2

6. Camacho A. Cholera epidemic in Yemen, 2016-18: an analysis of surveillance data. Lancet Glob Health. 2018 Jun; 6(6): e680-e690. doi: 10.1016/S2214-109X(18)30230-4

7. Ali M, Nelson AR, Lopez AL, Sack DA (2015) Updated Global Burden of Cholera in Endemic Countries. PLoS Negl Trop Dis 9(6): e0003832. https://doi.org/10.1371/journal.pntd.0003832

8. WHO (2014) Cholera surveillance and number of cases. Geneva: World Health Organization.

9. Masuet Aumatell C, Ramon Torrell JM, Zuckerman JN (2011) Review of oral cholera vaccines: efficacy in young children. Infection and drug resistance 4: 155-160. pmid:22114507

10. Griffith DC, Kelly-Hope LA, Miller MA (2006) Review of reported cholera outbreaks worldwide, 1995 2005. Am J Trop Med Hyg 75: 973-977. pmid:17123999

11. Zuckerman JN, Rombo L, Fisch A (2007) The true burden and risk of cholera: implications for prevention and control. Lancet Infect Dis 7: 521-530. PMID:17584531

12. Luquero FJ, Rondy M, Boncy J. Mortality rates during cholera epidemic, Haiti, 2010-2011. Emerg Infect Dis. 2016; 22: 410-416

13. Ali M, Lopez AL, You YA, Kim YE, Sah B, et al. (2012) The global burden of cholera. Bulletin of the World Health Organization 90: 209-218A. pmid:22461716 